\title{
1.2 .4 SCATTERING LAYER OF COSMIC DUST IN THE UPPER ATMOSPHERE
}

\author{
François Link \\ Institut d'Astrophysique \\ 75014 Paris, France
}

The upper atmosphere acts as a giant collector of cosmic dust swept by the Earth on its travel through space. The transient presence of cosmic dust in the upper atmosphere was detected by the rise of the luminance of the twilight sky during the activity of several meteoric showers either by balloon photometers launched by C.N.E.S. at Aire sur l'Adour (Fehrenbach et al., 1972 a) or by the similar photometer at the Pic du Midi Observatory ( Link, 1973).

The permanent presence of cosmic dust in the upper atmosphere is more difficult to prove. We introduced therefore a new technique of measurements by balloon photometers which enables us to obtain during the same ascent the twilight curves at small (30\%) and at large $\left(85^{\circ}\right)$ zenithal distances (Fehrenbach and Link, 1974). We can write for the luminances

$$
\begin{array}{ll}
b=R+r+M & \text { at zenithal distance } z=30^{\circ} \text { and } \\
b=R^{\prime}+r^{\prime}+M^{\prime} & \text { at zenithal distance } z^{\prime}=85^{\circ},
\end{array}
$$

where $R, R^{\prime}$ stand for the computed primary components, $r, r^{\prime}$ for the multiple scattering components and $M, M^{\prime}$ for the dust components measured at solar depressions $U$ and $U^{\prime}$.

Both twilight curves $\log b(U)$ and $\log b^{\prime}\left(U^{\prime}\right)$ were obtained for the ascent on June 29 th 1973 at $35 \mathrm{~km}$ i.e. outside any important shower activity (Fig.1). In the same graph are drawn the computed curves $\log R(U)$ and $\log R^{\prime}\left(U^{\prime}\right)$ according to Link and Weill (1975).

On both curves of $R$ and $R^{\prime}$ we pick up the points $A$ and $A^{\prime}$ where $R=R^{\prime}$ at solar depressions $U$ and $U^{\prime}$ ' and we compare there the 
observed luminances $b$ and $b^{\prime}$. We find $b^{\prime}>b$. As according to (1)

$$
b^{\prime}-b=M^{\prime}-M+r^{\prime}-r
$$

this excess of light near the horizon might be due either to the meteoric component $M^{\prime}$ or to the multiple scattering component $r^{\prime}$. As the latter is concerned, our method (Fehrenbach et al., 1972 b) based on azimuthal profile gives $r^{\prime} / R^{\prime}<0,1$ and the meteoric origin of the light excess can be accepted.

Now two effects may be the origin of it:

1) The shape of the scattering indicatrix of dust particles and

2) their stratification in the upper atmosphere.

In the first eventuality if the sedimentation of cosmic

dust in the upper atmosphere were governed only by the gravitation and the resistance of the quiet atmosphere, the concentration of the dust particles should be proportional to the number density of the air ( Link, 1950) which enters in both integrals of $R$ and $R^{\prime}$. In consequence

$$
M=c(\gamma) R \text { and } M^{\prime}=c\left(\gamma^{\prime}\right) R^{\prime}=c\left(\gamma^{\prime}\right) R
$$

where $c\left(\gamma^{\prime}\right)>c(\gamma)$ are the function of both scatterings angles $\gamma$ and $\gamma^{\prime}$. Therefore the ratio obtained at different solar depressions

$$
\frac{b^{\prime}-b}{b}=\frac{c\left(\gamma^{\prime}\right)-c\left(\gamma^{\prime}\right)}{1+c(\gamma)}=B
$$

should be nearly constant.

Actually we find for $\beta$ a curve (Fig.2) with a maximum near $U^{\prime}=14^{\circ}$. At this moment the edge of the Earth's shadow on the line of sight passes near the $110 \mathrm{~km}$ level (Fig.2). We can interpret it as a consequence of the stratification of the scattering medium at this level.

We reach a similar conclusion for the azimuthal profiles of the sky obtained at $z=85^{\circ}$ during the same ascent i.e.

$$
\begin{aligned}
& B(a)=b(a) / b\left(0^{\circ}\right) \text { compared with the theoretical profile } \\
& F(a)=R(a) / R\left(0^{\circ}\right)
\end{aligned}
$$

where $a$ is the azimuth computed from solar vertical plane where $a=0^{\circ}$. For the ratio of both we obtain with the aid of (1)

$$
k=\frac{B(a)}{F(a)}=\frac{1+M(a) / R(a)}{1+M\left(0^{\circ}\right) / R\left(0^{\circ}\right)}
$$

In this manner we obtained the curves of $k$ represented on Fig.3. The ratio $k$ rises or falls with the azimuth a if

$$
\mathrm{dk} / \mathrm{da} \gtrless 0
$$




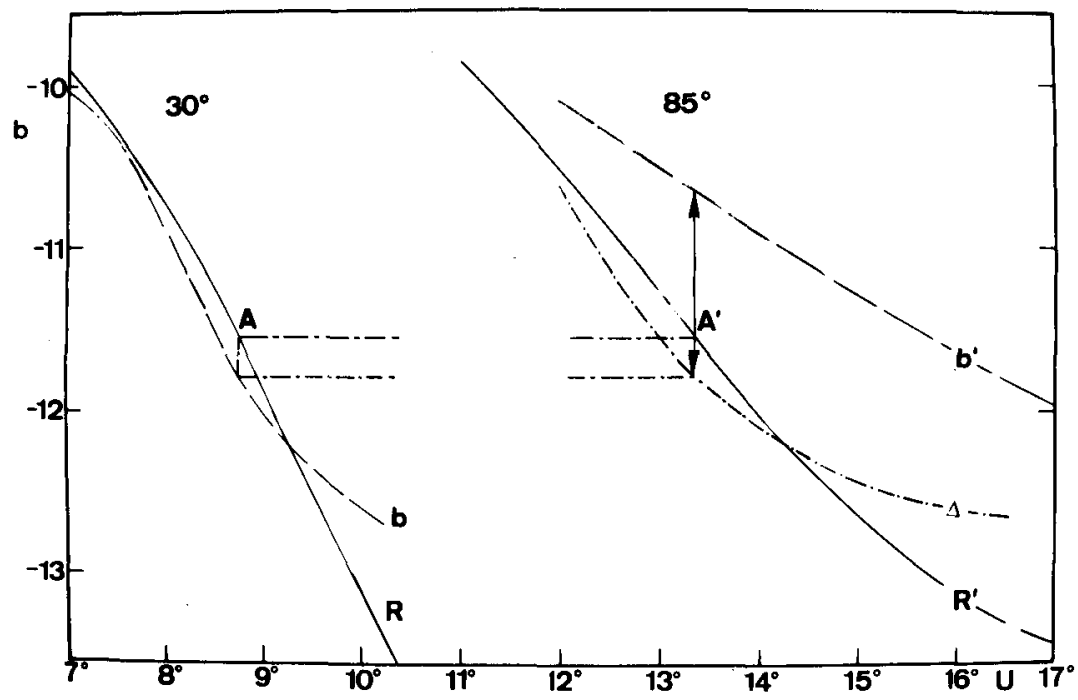

Fig. 1. Twilight curves at small $\left(30^{\circ}\right)$ and large $\left(85^{\circ}\right)$ zenithal distances in solar vertical $\left(a=0^{\circ}\right)$ for balloon ascent in June 1973 at Aire sur 1'Adour.

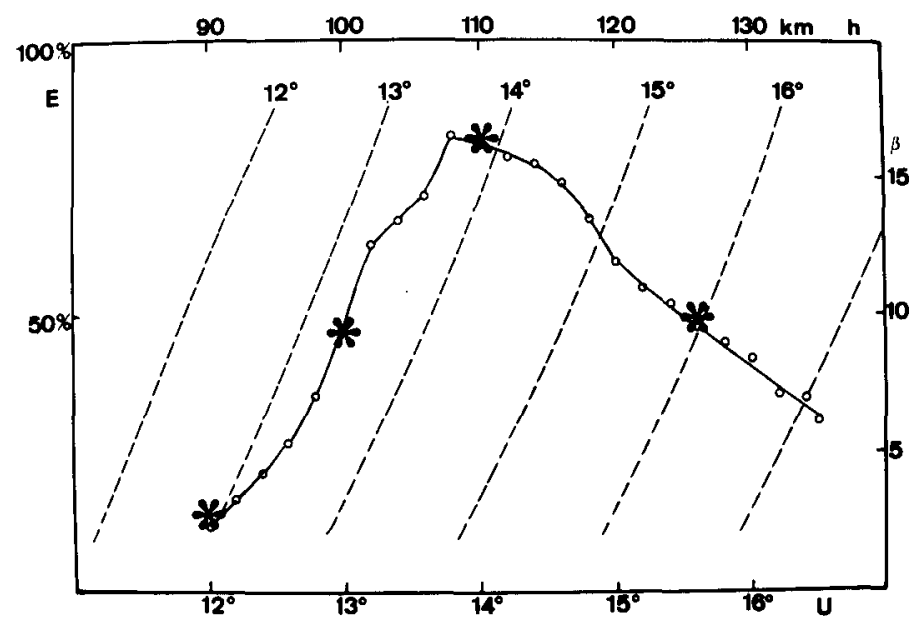

Fig.2. Curve of $\beta(-)$ as function of solar depression U. Curves (-- ) of solar illumination $\mathrm{E}$ for solar depressions $12^{\circ}-15^{\circ}$ as function of the height $h$ on the line of sight $z=85^{\circ}$ and $a=0^{\circ}$. Asterisks are the values of $\beta$ computed for a model of a homogeneous scattering layer between 110 and $130 \mathrm{~km}$. 
or if

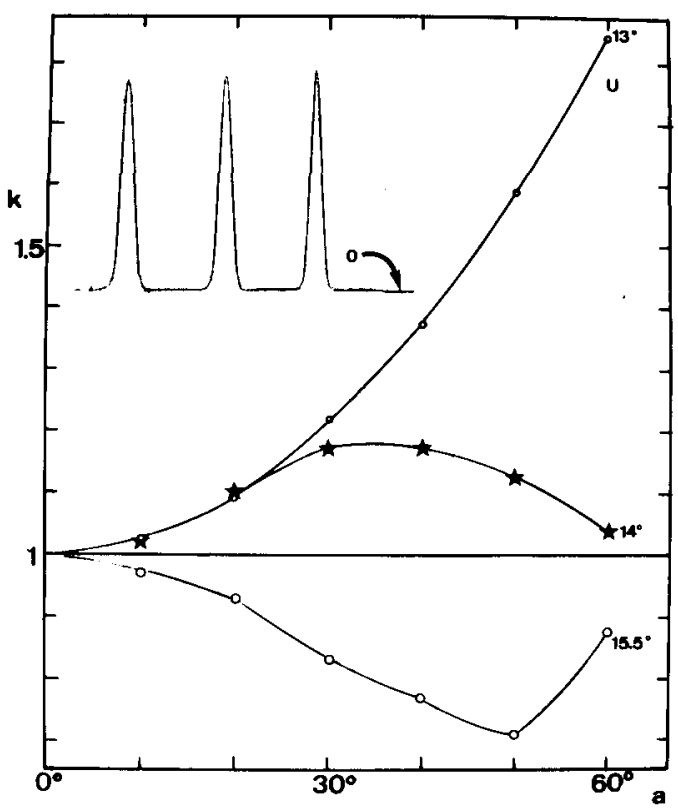

Fig.3. Curves of the variation of $k$ with the azimuth a for different solar depressions U. Inset : Specimen of the azimuthal profile b(a) at $z=85^{\circ}$; for a) $60^{\circ}$ the intensity falls practically to zero (obscurity) marked on the left by an arrow. The smallness of $r^{\prime}$ is therefore obvious.

$$
\mathrm{d} \log \mathrm{M} / \mathrm{da} \gtrless \mathrm{d} \log \mathrm{R} / \mathrm{da}
$$

Returning provisorily to the assumption (3) we get for (9)

$$
a \log c(\gamma) / d a \gtrless 0
$$

as the condition of rising or falling $k$ with a.

As a matter of fact in the interval $10^{\circ}<\gamma<70^{\circ}$ concerned by our measurements the above ratio remains constantly (Giese,1970)

$$
d \log c(\gamma) / d a<0
$$

and there cannot exist the rise of $\mathrm{k}$. On the curve of $\mathrm{k}$ at $U=14^{\circ}$ the fall starts at about $a=30-40^{\circ}$ and at this moment the shadow passes near the $120 \mathrm{~km}$ level. Below this level where $\mathrm{k}$ rises the condition (3) cannot be fulfilled and the stratification of the scattering medium takes place.

Summing up we reach the following conclusions :

a) The excess of light of the twilight sky near the horizon reveals the permanent presence of cosmic dust ( outside the activity 
of major meteoric showers) in the upper atmosphere.

b) Its distribution is different from the gravitational sedimentation.

c) The level $110-120 \mathrm{~km}$ is critical for this distribution.

It was suggested (Burnett, Lillie) at the presentation of this paper at the Heidelberg IAU Colloquium that the airglow continuum can intervene in our observations. This possibility demands a detailed discussion regarding the amount of airglow continuum and the much larger twilight luminance. Also the expected results of recent launching during ( 5.V.1975) and after (9.v.1975) the activity of etaAquarids may throw some light on to our problem.

\section{REFER E N C ES.}

Fehrenbach,M. ,Frimout,D.,Link,F. and Lippens,C. 1972 a, Ann. Géophys. 28,363

Fehrenbach,M. ,Frimout, D. ,Link,F.,Lippens, C. and Weill,G. 1972 b, Compt. Rendus. Acad. Sci. Paris B-275,223.

Fehrenbach,M. and Link,F. 1974, Compt. Rend. Acad. Sci. Paris B-279, 687

Giese, R.H. 1970, Max Planck Inst. PAE/Extraterr. 70, München

Link,F. 1950, Bull. Astron. Inst. Czechoslov.2, l

Link,F. 1973, Space Research XIV, 703

Link,F. and Weill,G. submitted for publication to Geophys. Ann. 